\title{
Research of Dual-channel Supply Chain Decision with Consideration of Low-carbon and Fairness Concerns
}

\author{
Guoshuai Niu ${ }^{1, *}$, Qi Wei ${ }^{1}$ \\ ${ }^{1}$ School of Business Administration, Guangdong University of Finance \& Economics, 21 Luntou Road, Guangzhou, 510320, China
}

\begin{abstract}
With in-depth implementation of policies on energy conservation and emission reduction and rising of low-carbon production practice in enterprises, low-carbon supply chain has gradually become a research issue which causes extensive attentions from scholars. However, most existing researches focus on supply chain decision and coordination under the background of single channel or mere consideration of fairness concerns of retailers. Therefore, the fairness concerns of manufacturers were taken into account in the double-channel low-carbon supply chain. A double-channel low-carbon supply chain model was constructed to research the influence of manufacturers' fairness concerns to the optimal decision-making of all parties in the supply chain and to the overall performance of supply chain. The research results suggested that: with improvement of manufacturers' fairness concern coefficient, the decision-making of supply chain members demonstrated different changing strength; the manufacturers' fairness concern caused harm to overall profit of supply chain.
\end{abstract}

\section{Introduction}

In recent years, global environmental problems have become increasingly intensified. Energy conservation and emission reduction have become the consensus and inevitable trend of the international community. Countries around the world have put forward emission reduction targets. In order to achieve emission reduction targets, countries have adopted different measures, such as levying carbon taxes, promoting clean energy and Low-carbon technology, elimination of low-capacity and highemission enterprises. At the same time, consumers' awareness of low-carbon environmental protection is also increasing. Studies have shown that consumers are more willing to pay for low-carbon products and are willing to pay higher prices for low-carbon products. Under the influence of relevant policies and increased consumer awareness of environmental protection, upstream manufacturers have put forward low-carbon transformation strategies to produce low-carbon products. For example, the world-renowned mobile phone manufacturer Apple is committed to reducing the carbon footprint of its products through technological innovation. Since 2008, the average energy consumption of Apple products has been reduced by $73 \%$. Reducing product packaging is expected to reduce carbon emissions by more than 2 million tons per year. Benjaafar S first incorporated carbon emission factors into the supply chain decisionmaking system, and used the classic batch model to study how carbon emission factors affect the decision-making of supply chain members ${ }^{[1]}$. Subsequently, many scholars conducted extensive research on low-carbon supply chain.
Motoshita M et al. studied the issue of consumers' lowcarbon preference and found that consumers have a clear preference for low-carbon products, and are even willing to pay higher prices for low-carbon products ${ }^{[2]}$

The low-carbon level of products in the supply chain system is mainly determined by the manufacturer. Therefore, in the context of energy saving and emission reduction, whether it is the transformation and upgrading of production technology or the promotion of low-carbon products, this requires manufacturers to invest a lot of costs. This is bound to affect the profit level of manufacturers, intensify conflicts between manufacturers and retailers, and is not conducive to supply chain coordination. Cui et al. introduced fairness concerns into traditional channels, and the study found that manufacturers can achieve supply chain coordination by setting wholesale prices higher than marginal costs ${ }^{[3]}$. At the same time, due to the maturity of e-commerce technology and the continuous improvement of logistics networks, more and more consumers have become accustomed to online shopping, prompting manufacturers to open up online direct sales channels on the basis of traditional retail channels. This is affected by factors such as product services and convenience. As a result, the emergence of online direct sales channels will inevitably cause conflicts and competition between channels ${ }^{[4]}$. Under the influence of energy-saving emission reduction and dual-channel sales model, the competition conflicts between manufacturers and retailers and the manufacturers' carbon emission reduction decisions have become issues worthy of study.

* Corresponding author: 398705038@qq.com 


\section{Model Settings}

Assume that there is only one manufacturer and one retailer in the dual-channel green supply chain system, where the manufacturer produces a single low-carbon product and sells its products through the retailer's traditional channels and self-developed online direct sales channels. The notation for the model is summarized in Table 1.

The function of the manufacture's carbon abatement cost and the product's low- carbon level is

$$
I=\frac{1}{2} \beta e^{2}
$$

The demand functions of low-carbon products in the two channels are:

$$
\begin{gathered}
D_{r}=v-p_{r}+b p_{m}+e \\
D_{m}=1-v-p_{m}+b p_{r}+e
\end{gathered}
$$

Manufacturer's profit, retailer's profit and total supply chain profit is given by:

$$
\begin{gathered}
\pi_{m}=w D_{r}+p_{m} D_{m}-\frac{1}{2} \beta e^{2} \\
\pi_{r}=\left(p_{r}-w\right) D_{r} \\
\pi_{s c}=\pi_{m}+\pi_{r}=p_{r} D_{r}+p_{m} D_{m}-\frac{1}{2} \beta e^{2}
\end{gathered}
$$

Table1. Summary of Model Variables

\begin{tabular}{ll}
\hline Variable & \\
\hline$w$ & Unit product wholesale price; $\mathrm{w} \geq 0$ \\
$e$ & Low-carbon level; $\mathrm{e} \geq 0$ \\
$p_{r}$ & Retail price of traditional channels; $\mathrm{p}_{\mathrm{r}} \geq 0$ \\
$p_{m}$ & Retail price of direct sales channel; $\mathrm{p}_{\mathrm{m} \geq 0}$ \\
& \\
$I$ & The emission reduction cost; $\mathrm{I} \geq 0$ \\
$D_{r}$ & Market demand for traditional channels \\
$D_{m}$ & Market demand for online direct sales channels \\
& \\
$\pi_{r}$ & Retailer's profit \\
$\pi_{m}$ & Manufacturer's profit \\
$\pi_{s c}$ & Overall supply chain profit \\
& \\
$a$ & Basic market demand; $\mathrm{a}=1$ \\
$v$ & Market share of traditional channels $; 0<\mathrm{v}<1$ \\
$b$ & Cross price influence coefficient; $\mathrm{b}>0$ \\
$\lambda$ & Manufacturer's fairness concern coefficient \\
$\beta$ & Low-carbon product abatement cost coefficient \\
$c$ & Unit product production cost; $\mathrm{c}=0$
\end{tabular}

\section{Model solving}

\subsection{Decentralised model without fairness concerns}

In the decentralized model, both supplier and retailer seek to maximize their own profits, and a Stackelberg game is played between the two parties, that is, the manufacturer first determines the wholesale price and online retail price of the product, and then the retailer determines its retail price.

The equilibrium solutions are gained through the backward induction method.

The optimal decisions are obtained as follows:

$$
\begin{gathered}
{p_{m D}}^{*}=\frac{4 \beta(v-b v-1)-2 v+1}{2(1+b)(b-4 \beta+4 \beta b+3)} \\
w_{D}^{*}=\frac{4 \beta(b v-b-v)+4 v+2 b v-b-2}{2(1+b)(b-4 \beta+4 \beta b+3)} \\
e_{D}^{*}=\frac{v-b v-2}{b-4 \beta+4 \beta b+3} \\
p_{r D}^{*}=\frac{v\left(4 b+4 \beta b+2 \beta b^{2}+6-6 \beta\right)-2 b-4 \beta b-3}{2(1+b)(b-4 \beta+4 \beta b+3)}
\end{gathered}
$$




\subsection{Decentralised model with fairness concerns}

In the decentralized model, manufacturers' fairness concerns are introduced, that is, manufacturers not only pay attention to their own interests, but also measure the fairness of channel profit distribution. Assume that the decision makers are equally sensitive to the same profit and $\operatorname{loss}^{[5]}$. the manufacturer's utility function can be obtained as

$$
U_{m}=\pi_{m}-\lambda\left(\pi_{r}-\pi_{m}\right)
$$

The equilibrium solutions are gained through the backward induction method.

The optimal decisions are obtained as follows:

$$
p_{m \lambda}{ }^{*}=\frac{\lambda(6 \beta v-6 \beta-2 v-6 \beta b v+1)+4 \beta(v-b v-1)-2 v+1}{2(1+b)(b-4 \beta+4 \lambda+4 \beta b-6 \beta \lambda+2 b \lambda+6 \beta b \lambda+3)}
$$

$$
\begin{aligned}
& e_{\lambda}^{*}=\frac{\lambda(2 v-2 b v-3)+v-b v-2}{b-4 \beta+4 \lambda+4 \beta b-6 \beta \lambda+2 b \lambda+6 \beta b \lambda+3} \\
& p_{r \lambda}^{*}=\frac{\lambda\left(10 v+8 b v+6 \beta b v+4 \beta^{2} b v-4 b-6 \beta b-10 \beta v-5\right)+2 v\left(2 b+2 \beta b+\beta b^{2}-3 \beta+3\right)-2 b-4 \beta b-3}{2(1+b)(4-4 \beta+4 \lambda+4 \beta b-6 \beta \lambda+2 b \lambda+6 \beta b \lambda+3)}
\end{aligned}
$$

\section{Analysis}

In this section, we analyze the optimal results obtained by modeling in order to obtain some meaningful conclusions and enlightenments for business practice.

Proposition 4.1: $w_{\lambda}{ }^{*}, p_{r \lambda}{ }^{*}$ are positively related with $\lambda$.

The proof of Proposition 4.1 is given in Appendix 1 .

As indicated in Proposition 4.1, In a dual-channel supply chain dominated by manufacturers, the fairness concerns of manufacturers will lead to the redistribution of supply chain profits. Because the production of lowcarbon products increases production costs, manufacturers will naturally set higher wholesale prices for retailers, and retailers have to passively accept higher wholesale prices due to their weaker binding force on manufacturers, and at the same time, in order to ensure themselves profits increase offline retail prices.

Proposition 4.2: $e_{\lambda}^{*}$ are negatively related with $\lambda$.

The proof of Proposition 4.2 is given in Appendix 2.

Proposition 4.2 shows, in order to obtain more profits, manufacturers will appropriately lower online direct selling prices to gain a higher market share, and under the constraints of relevant policies, reduce the low-carbon level of products as much as possible to reduce production costs.

Proposition 4.3: $\pi_{s c \lambda}{ }^{*}$ is negatively related with $\lambda$.

The method of proof is the same as Proposition 4.1.

Proposition 4.3 states that the manufacturer's concerns about fairness will harm the profits of the supply chain. As the leader of the supply chain, they should adopt a cooperative attitude, try to reduce their own fairness concern coefficient, and strive to maintain fairness and neutrality.

\section{Conclusion}

In the context of the dual-channel low-carbon supply chain, we consider the fairness concerns of manufacturers and discusses the impact of the optimal decision-making of supply chain members and the fairness concerns of suppliers on the low-carbon level of products, retail prices, and profit distribution. Research indicates: (1) As the manufacturer's fairness concern coefficient increases, the decision-making of supply chain members presents different trends. Among them, the manufacturer's online direct selling price decreases with the increase of the fairness concern coefficient. This is because the manufacturer wants to get more Market share in order to further enhance its own profits. (2) The fairness concerns of manufacturers will harm the overall profits of the supply chain. As the leader of the supply chain, suppliers should try to reduce their own fairness concerns and strive to maintain fairness and neutrality. (3) Comparing the three decision-making modes, it is found that the lowcarbon level of products is the highest under the centralized decision-making mode, while the decentralized decision-making fairness and neutral mode will be the next time the product is the lowest when the manufacturer is fair. In the future, we can further study the impact of the information asymmetry strategy of manufacturers and retailers on fairness concerns on the optimal decision-making and profit of the supply chain.

\section{Appendices}

Appendix 1. Proof of Proposition 4.1

Proof

$\frac{\partial w_{\lambda}{ }^{*}}{\lambda}=\frac{(b-4 \beta+4 \beta b+4)(2 v-2 \beta v+2 \beta b v-1)}{2(b-4 \beta+4 \lambda+4 \beta b-6 \beta \lambda+2 b \lambda+6 \beta b \lambda+3)^{2}}$ 
$\frac{\partial p_{r \lambda}{ }^{*}}{\partial \lambda}=\frac{4(b-1)(b-3 \beta+3 \beta b+2)(2 v-2 \beta v+2 \beta b v-1)}{(b-4 \beta+4 \lambda+4 \beta b-6 \beta \lambda+2 b \lambda+6 \beta b \lambda+3)^{3}}$

since each variable is greater than 0 , and $0<\mathrm{b}<1, \beta b-\beta+1$

$<0$, so

$$
\frac{\partial w_{\lambda}{ }^{*}}{\lambda}>0, \frac{\partial p_{r \lambda}{ }^{*}}{\partial \lambda}>0
$$

Appendix 2. Proof of Proposition 4.2

Proof

$\frac{\partial p_{m \lambda}{ }^{*}}{\partial \lambda}=\frac{2 v(\beta b-\beta+1)-1}{2(b-4 \beta+4 \lambda+4 \beta b-6 \beta \lambda+2 b \lambda+6 \beta b \lambda+3)^{2}}$

$\frac{\partial e_{\lambda}^{*}}{\lambda}=\frac{(1-b)(2 v-2 \beta v+2 \beta b v-1)}{2(b-4 \beta+4 \lambda+4 \beta b-6 \beta \lambda+2 b \lambda+6 \beta b \lambda+3)^{2}}$

since each variable is greater than 0 , and $0<\mathrm{b}<1, \beta b-\beta+1$

$<0$, so

$\frac{\partial p_{m \lambda}{ }^{*}}{\partial \lambda}<0, \frac{\partial e_{\lambda}^{*}}{\lambda}<0$

\section{References}

1. S.Benjaafar,Y.Li,M.Daskin,IEEE.T.Autom.Sci.Eng,1 0,99 (2013)

2. M.Motoshita, M.Sakagami, Y.Kudoh, K.Tahara, A.Inaba, J.Clean. Prod ,101,205 (2015);

3. H.Cui,T.Raju,Z.Zhang,Manage.Sci,53,1303(2007)

4. J.Ji,Z.Zhang,L.Yang,J.Clean.Prod,141,852 (2017)

5. S.Du,T.Nie,C.Chu,Int.J,Prod.Res,52,5070 (2014) 